\title{
Hydrogen-rich saline alleviates early brain injury through inhibition of necroptosis and neuroinflammation via the ROS/HO-1 signaling pathway after traumatic brain injury
}

\author{
YUN HU, XIAOYAN FENG, JUNHUI CHEN, YAN WU and LIUYAN SHEN \\ Department of Neurosurgery, Wuxi Clinical College of Anhui Medical University, \\ 904th Hospital of Joint Logistic Support Force of PLA, Wuxi, Jiangsu 214044, P.R. China
}

Received September 6, 2021; Accepted November 9, 2021

DOI: $10.3892 /$ etm.2021.11049

\begin{abstract}
Traumatic brain injury (TBI) has been recognized as a serious public health issue and a key contributor to disability and death, with a huge economic burden worldwide. Hydrogen, which is a slight and specific cytotoxic oxygen radical scavenger, has been demonstrated to ameliorate early brain injury (EBI) through reactive oxygen species (ROS), oxidative stress injury, apoptosis and necroptosis. Necroptosis refers to a type of programmed cell death process that has a vital function in neuronal cell death following TBI. The specific function of necroptosis in hydrogen-mediated neuroprotection after TBI, however, has yet to be determined. The present study aimed to examine the neuroprotective effects and possible molecular basis that underly hydrogen-rich saline in TBI-stimulated EBI by examining neural necroptosis in the C57BL/6 mouse model. The brain water content, neurological score, neuroinflammatory cytokines (NF- $\kappa \mathrm{B}, \mathrm{TNF}-\alpha, \mathrm{IL}-6$ and IL-1 $\beta$ ) and ROS were evaluated using flow cytometry. Malondialdehyde, superoxide dismutase (SOD) and glutathione (GSH) levels were evaluated using a biochemical kit. Receptor-interacting protein kinase (RIP)1, RIP3, Nrf2 and Heme oxygenase-1 (HO-1) were evaluated using western blotting. mRNA of Nrf2 and HO-1 were evaluated using quantitative PCR. Neuronal death was evaluated by TUNEL staining. The outcomes illustrated that hydrogen-rich saline treatment considerably enhanced the neurological score, increased neuronal survival, decreased the levels of serum MDA and brain ROS, increased the levels of serum GSH and SOD. In addition the protein expression levels of RIP1 and RIP3 and the cytokines NF- $\kappa$ B, TNF- $\alpha$, IL-1 $\beta$ and IL- 6 were downregulated compared with the TBI
\end{abstract}

Correspondence to: Dr Junhui Chen, Department of Neurosurgery, Wuxi Clinical College of Anhui Medical University, 904th Hospital of Joint Logistic Support Force of PLA, 101 Xing Yuan North Road, Wuxi, Jiangsu 214044, P.R. China

E-mail: chenjunhui101@163.com

Key words: hydrogen-rich saline, traumatic brain injury, early brain injury, necroptosis, neuroinflammation group, which demonstrated that hydrogen-rich saline-induced inhibition of necroptosis and neuroinflammation ameliorated neuronal death following TBI. The neuroprotective capacity of hydrogen-rich saline was demonstrated to be partly dependent on the ROS/heme oxygenase-1 signaling pathway. Taken together, the findings of the present study indicated that hydrogen-rich saline enhanced neurological outcomes in mice and minimized neuronal death by inducing protective effects against neural necroptosis as well as neuroinflammation.

\section{Introduction}

Traumatic brain injury (TBI) has been recognized as a serious public health issue and a key contributor to disability and death, with a huge economic burden worldwide $(1,2)$. High incidence (1/1,000 in China between 1983 and 1985) of TBI is predominantly reported in low- and middle-income countries as well as developing countries, including China and Iran (1-3). The prevalence of TBI has witnessed a rapidly growing trend due to the considerable increase in road accidents, such as motor vehicle collisions (2). Although an increasing number of randomized controlled trials have included intracranial pressure monitoring, therapeutic hypothermia, surgical methods and drug administration in recent years, long-term outcomes have not substantially improved, especially after drug intervention (2-8). Therefore, it is important to further clarify the physiopathological processes of TBI and identify novel efficient pharmacological targets for TBI treatment. It is widely acknowledged that the pathophysiology of TBI encompasses several types of pathological and physiological changes, mainly involving primary brain injury and secondary brain injury, which leads to neuronal death, neurological deficits and mortality after TBI (9). Primary brain injury, which is a direct physical injury to the brain tissue, is difficult to prevent and cannot be usually reversed, and leads to brain tissue disorganization, intracerebral hemorrhage and blood-brain barrier (BBB) damage $(1,10,11)$. Secondary brain injury includes calcium overload, oxidative stress, neuroinflammation, autophagy, lipid peroxidation, apoptosis and necroptosis, and can be reversed $(10,11)$.

Necroptosis has recently been identified as a pathway of modulated necrosis and a mechanism of caspase-independent 
programmed cell death, requiring the proteins mixed lineage kinase domain-like (MLKL) and receptor-interacting protein kinase-3 (RIP3), and is triggered by death receptors (12). Accumulating evidence indicates that necroptosis performs an important function in central nervous system disorders, such as TBI (13-15), intracerebral hemorrhage (16,17), ischemic stroke (18), Alzheimer's disease, Parkinson's disease and amyotrophic lateral sclerosis (19). The activation of a TNF ligand family member, such as RIP1 and MLKL, is the most upstream signaling activity necessary for necroptosis induction $(20,21)$. The activation of RIP1 contributes to necroptosis via inducting RIP1-RIP3-MLKL complex (22). Necroptosis is common in early brain injury (EBI) and may be a mechanism of TBI.

In previous years, it has become widely acknowledged that hydrogen-rich saline or hydrogen gas can protect the human body from numerous diseases, such as neurodegenerative disorders, spontaneous subarachnoid hemorrhage, stroke and ischemia-reperfusion damage, by modulating neuronal apoptosis, inflammatory response and oxidative stress (23-26). A previous study has indicated that hydrogen may selectively minimize peroxynitrites and hydroxyl radicals, and subsequently have a crucial function exhibiting cytoprotective, anti-inflammatory, anti-apoptotic and antioxidant properties (27). However, the neuroprotective benefits of hydrogen-rich saline treatment on TBI are controversial. Heme oxygenase-1 (HO-1) is an essential element of the cellular defense system that is activated by oxidant-stimulated damage and acts against it $(28,29)$. In the central nervous system (CNS), HO-1 exerts anti-necroptotic, anti-neuroinflammatory and neuroprotective functions $(27,30)$. In our previous study, HO-1 was demonstrated to regulate neuronal death in acute CNS disease (31). Therefore, treatments that target HO-1 may be potentially effective at preventing necroptosis, oxidative stress and inflammation following TBI. Nonetheless, the specific mechanisms regarding the potential neuroprotective benefits of hydrogen-rich saline treatment are unknown. The present study examined whether neuroprotective benefits of hydrogen-rich saline treatment in a mouse model of TBI occur via effects on neuroinflammation and necroptosis, and whether neuroprotection is dependent on the reactive oxygen species (ROS)/HO-1 pathway.

\section{Materials and methods}

Animals. All the animal experiments conducted in the present study were completed in compliance with guidelines for the appropriate handling of laboratory animals formulated by the National Institutes of Health (32). Approval of the experiments was provided by the Ethics Committee of Wuxi Clinical College of Anhui Medical University (Wuxi, China). A total of 36 healthy adult male C57BL/6J mice (age, 8-10 weeks; Anhui Medical University, Hefei, China) weighing between 22-25 g were used when conducting all the experiments for the current study. The mice were maintained in animal care facilities (temperature, $25 \pm 2^{\circ} \mathrm{C}$; humidity, $55 \pm 5 \%$ ) with a $12 / 12 \mathrm{~h}$ dark/light cycle and an unrestricted supply of water and food.

Animal TBI model. The Feeney weight-drop model of focal injury was strictly followed when developing the TBI model $(33,34)$. Briefly, anesthetization of the mice was performed using $1 \%$ sodium pentobarbital $(40 \mathrm{mg} / \mathrm{kg})$ injected intraperitoneally. The mice were subsequently placed in a brain stereotaxic apparatus. While being operated, a heating pad was utilized to ensure that the rectal temperature remained at $37 \pm 0.5^{\circ} \mathrm{C}$. The coordinates utilized when making a burr hole in the left hemisphere were set as follows: $0.2 \mathrm{~mm}$ posterior, $1 \mathrm{~mm}$ lateral and $2.2 \mathrm{~mm}$ ventral of bregma's horizontal plane. To visualize the dura mater, the bone fap was removed. The dura was subsequently placed under weight-drop equipment that included an impact sensor pedal; (Anhui Zhenghua Biological Instrument Equipment Co., Ltd.). Metal with a tip diameter of 3-mm and weight of $240 \mathrm{~g}$ was dropped onto the dura mater from a distance of $1 \mathrm{~cm}$ above the dura via a catheter. The scalp was subsequently closed and the mice were removed from the stereotaxic apparatus. Subsequently, a medical bone wax was utilized to cover the hole. The animals in the sham cohort received comparable surgical treatments but without the weight-drop procedure. At $72 \mathrm{~h}$ following TBI, the mice were sacrificed with $100 \mathrm{mg} / \mathrm{kg}$ sodium pentobarbital via an intraperitoneal injection. Brain tissue samples were collected after the mice were sacrificed. Fresh specimens (cerebral cortex) and serum were stored in liquid nitrogen $\left(-196^{\circ} \mathrm{C}\right)$ or hippocampus was stored in $4 \%$ formalin-fixed $\left(4^{\circ} \mathrm{C}\right)$ for $\geq 48 \mathrm{~h}$.

Drug preparation and administration. After the TBI model was established successfully, the mice received intraperitoneal injections every day for $72 \mathrm{~h}$ that contained either plain saline (control) or hydrogen-rich $(5 \mathrm{ml} / \mathrm{kg}$; experimental). Hydrogen-rich saline was prepared in accordance with the procedure described in previous studies $(35,36)$. Briefly, purified hydrogen was dissolved in normal saline for $2 \mathrm{~h}$ at an elevated pressure of $0.4 \mathrm{MPa}$. The physiological concentration was maintained at $1.73 \mathrm{ml}$ hydrogen per $100 \mathrm{ml}$ saline (average, $>6 \mathrm{mmol} / \mathrm{l}$ ). Hydrogen-rich saline was stored at $4^{\circ} \mathrm{C}$ in an aluminum bag without dead volume under atmospheric pressure. Every week, fresh hydrogen-rich saline was synthesized to guarantee a consistent concentration. The content of hydrogen in saline was evaluated and detected by gas chromatography as reported in a previous study (37).

Neurobehavioral assessment. By employing a previously published neurological grading system $(38,39)$, the degree of brain injury was assessed $72 \mathrm{~h}$ following TBI via the determination of neurological function. The scoring system consisted of reflex, sensory, balance and motor tests. The neurological scores ranged between 0 and 18 and were calculated by summing the individual scores (Table SI). A behavioral assessment was conducted on all mice in each cohort, with a higher score indicating impaired neurological function.

Brain water-content measurement. As reported previously, the degree of brain edema was examined by quantifying the brain water content utilizing the standard wet-dry technique (39-41). A total of $72 \mathrm{~h}$ following TBI the mice were euthanized, and their whole brain was extracted and divided into the contralateral and ipsilateral cortex, contralateral and ipsilateral basal ganglia and cerebellum (wet weight). The dry weight of each cohort's brain specimen was then determined after dehydrating each part at $105^{\circ} \mathrm{C}$ for $24 \mathrm{~h}$. The proportion of 
brain water content was equal to (wet weight-dry weight)/wet weight $\mathrm{x} 100 \%$.

Cytokine measurements. ELISA was utilized to measure cerebral cortex NF- $\kappa \mathrm{B}$ (cat. no. ab176663), cerebral cortex TNF- $\alpha$ (cat. no. ab208348), cerebral cortex IL-6 (cat. no. ab222503) and cerebral cortex IL-1 $\beta$ (cat. no. ab197742; all from Abcam). The cerebral cortex was extracted from mice brain. The procedure was conducted in accordance with the manufacturer's protocols.

Analysis of ROS. The brain tissue intracellularROS synthesis was evaluated utilizing the non-fluorescent diacetylated 2',7'-dichlorofluorescein diacetate (DCFH-DA) probe (Sigma-Aldrich; Merck KGaA) that becomes highly fluorescent upon oxidation and was used according to the guidelines provided by the manufacturer. Fresh cerebral cortex was used to form a single cell suspension. The cells were harvested at a concentration of $2 \times 10^{6}$ cells $/ \mathrm{ml}$, then DCFH-DA ( $10 \mu \mathrm{M}$ final concentration) was added and the mixture was incubated at $37^{\circ} \mathrm{C}$ for $15 \mathrm{~min}$. The DCFH results were examined via flow cytometry (CytoFLEX; Beckman Coulter, Inc.). CytExpert (version 2.4; Beckman Coulter, Inc.) was used to analyze the data.

Analysis of MDA, superoxide dismutase (SOD) and glutathione (GSH). The malondialdehyde (MDA) Assay Kit (excitation/emission, 532/553 nm; cat. no. ab118970; Abcam) was utilized to detect serum MDA levels in compliance with the instructions provided by the manufacturer. The serum was extracted from mice venous blood. The SOD Assay Kit (cat. no. A001-3-2; Nanjing Jiancheng Bioengineering Institute) and GSH (42) Assay Kit (cat. no. A005-1-2; Nanjing Jiancheng Bioengineering Institute) were utilized to detect serum SOD and serum GSH levels in compliance with the manufacturer's instructions $(43,44)$.

TUNEL staining. To evaluate neuronal death in the brain cortex, TUNEL staining was utilized. The procedure was performed with a TUNEL staining kit (cat. no. 11684817910; Roche Diagnostics $\mathrm{GmbH}$ ) according to the protocols provided by the manufacturer. In each $4 \%$ formalin-fixed $\left(4^{\circ} \mathrm{C}\right.$; duration, $\geq 48 \mathrm{~h}$ ) for specimen, paraffin-embedded sections $(10-\mu \mathrm{m})$ were cut from formalin-fixed tissue. Subsequently, $50 \mu 1$ TUNEL reaction mixture was added. The negative control used did not include the TUNEL reaction mixture. Subsequently, the slides were subjected to incubation in a humidified dark chamber at a temperature of $37^{\circ} \mathrm{C}$ for $60 \mathrm{~min}$. Then, a primary antibody against the neuronal marker NeuN (1:200; rabbit polyclonal; cat. no. ab128886; Abcam) diluted in PBS was added, followed by incubation overnight at $4^{\circ} \mathrm{C}$. Subsequently, incubated with FITC goat anti-mouse IgG secondary antibodies (1:100; cat. no. BA1101; Boster Biological Technology Co., Ltd.) at temperature of $37^{\circ} \mathrm{C}$ for $1.5 \mathrm{~h}$. Next, the slides were incubated at ambient temperature for $5 \mathrm{~min}$ in the dark with DAPI for staining of the nuclei, followed by imaging using a fluorescence microscope (magnification, x200). The validation of the cell count was performed using four high-power fields randomly selected, and the data obtained from each field were averaged.

Western blot analysis. Western blot analysis was conducted as indicated previously (40). Cerebral cortex specimens were collected and extracted. Cerebral cortex samples were collected, homogenized and total protein was extracted using RIPA buffer (CoWin Biosciences). The protein concentrations were quantified utilizing a BCA Protein Assay kit (Beyotime Institute of Biotechnology) and proteins (40 $\mu \mathrm{g} / \mathrm{lane})$ were separated using 10\% SDS-PAGE. Then, the proteins were transferred onto Immobilon nitrocellulose membranes. Blocking of the membranes was performed with $5 \%$ non-fat milk for $1 \mathrm{~h}$ at room temperature. The membranes were then subjected to incubation using the following primary antibodies overnight at $4^{\circ} \mathrm{C}$ : Anti- $\beta$-actin $(1: 1,000$; rabbit polyclonal; cat. no. ab8227; Abcam), anti-RIP1 (1:1,000; rabbit polyclonal; cat. no. ab106393; Abcam), anti-nuclear factor erythroid 2-related factor 2 (Nrf2; 1:1,000; rabbit polyclonal; cat. no. ab31163; Abcam), anti-HO-1 (1:1,000; rabbit polyclonal; cat. no. ab13243; Abcam) and anti-RIP3 (1:1,000; rabbit polyclonal; cat. no. ab62344; Abcam). After washing the membranes using $0.5 \%$ TBS-Tween-20 three times, the membranes were incubated with HRP-conjugated goat anti-rabbit IgG secondary antibodies (1:2,000; cat. no. 7074s; Cell Signaling Technology, Inc.) at room temperature for $1.5 \mathrm{~h}$. The signals were developed using an enhanced chemiluminescence reagent (MilliporeSigma) according to the manufacturer's instructions. A Bio-Rad imaging system (Bio-Rad Laboratories, Inc.) was utilized to identify protein bands that were then measured using ImageJ software (version 1.52; National Institutes of Health).

$R N A$ extraction and reverse transcription-quantitative $P C R$ $(R T-q P C R)$. TRIzol ${ }^{\circledR}$ reagent (Thermo Fisher Scientific, Inc.) was utilized to isolate total RNA from the cerebral cortex of brain specimens as per the guidelines provided by the manufacturer, which was then quantified utilizing a NanoDrop ${ }^{\mathrm{TM}} 2000$ spectrophotometer (Thermo Fisher Scientific, Inc.). The RevertAid First Strand cDNA Synthesis Kit (cat. no. K1622; Thermo Fisher Scientific Inc.) was subsequently utilized to perform reverse transcription of RNA to cDNA according to the manufacturer's protocol. Each specimen's mRNA levels were quantified via qPCR utilizing SYBR Green Master Mix (Toyobo Life Science). The expression levels of all genes were normalized to that of $\beta$-actin. The following qPCR thermocycling criteria were used: $45^{\circ} \mathrm{C}$ ( $2 \mathrm{~min}$ ) and $95^{\circ} \mathrm{C}(10 \mathrm{~min})$, followed by 40 cycles of denaturation at $95^{\circ} \mathrm{C}(15 \mathrm{sec})$, annealing at $60^{\circ} \mathrm{C}(1 \mathrm{~min})$ and extension at $72^{\circ} \mathrm{C}(1 \mathrm{~min})$. The analysis of all specimens was performed in triplicate. The primers used are listed as follows: Nrf2 forward, 5'-CAGTGCTCCTATGCGTGAA-3' and reverse, 5'-GCGGCTTGAATGTTTGTCT-3'; HO-1 forward, 5'-TGA CAGAAGAGGCTAAGACCG-3' and reverse, 5'-AGTGAG GACCCACTGGAGGA-3'; and GAPDH forward, 5'-ATG GGTGTGAACCACGAGA-3' and reverse, 5'-CAGGGATGAT GTTCTGGGCA-3' (45).

Statistical analysis. All experiments were performed with at least three experimental repeats, and the data are presented as the mean \pm SEM. Neurological scores are presented as the median and interquartile range. Statistical analyses were implemented using GraphPad Prism 6 (GraphPad Software, Inc.) and SPSS 14.0 (SPSS, Inc.). Differences between multiple groups were analyzed using one-way ANOVA followed by 

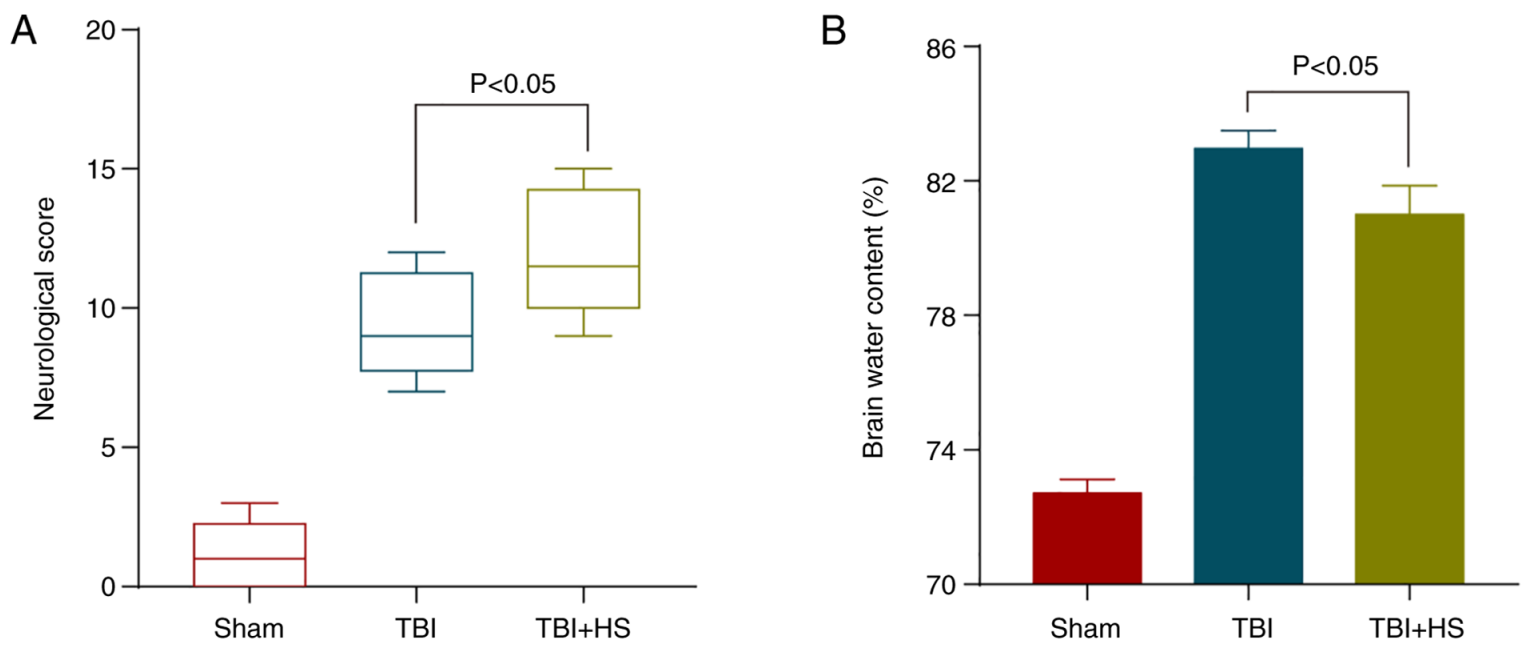

Figure 1. HS alleviates neurological impairment and brain edema after TBI. (A) Neurological scores of mice in the sham, TBI and TBI + HS cohorts $72 \mathrm{~h}$ after TBI (median and interquartile range; $\mathrm{n}=10$ ). (B) Brain water content in the three cohorts. Brain water content decreased significantly after HS treatment (mean \pm SEM; $n=5$ ). TBI, traumatic brain injury; HS, hydrogen-rich saline.

Tukey's post hoc test. Neurological scores were analyzed using a non-parametric test (Kruskal-Wallis followed by Dunn's post hoc test). $\mathrm{P}<0.05$ was considered to indicate a statistically significant difference.

\section{Results}

Hydrogen-rich saline alleviates neurological deficits and brain edema after TBI. Modified neurological severity scores were employed to assess neurological impairments to understand the neuroprotection of hydrogen-rich saline following TBI. To assess brain damage, the wet-dry technique was employed to measure brain water content $72 \mathrm{~h}$ after TBI. The findings illustrated that TBI was associated with a considerable increase in the brain water content, which was alleviated after hydrogen-rich saline treatment (Fig. 1B). Comparable findings in neurological scores indicated that the scores were considerably higher in animals suffering from hydrogen-rich saline treatment compared with the TBI group, and that hydrogen-rich saline treatment substantially improved the neurological function (Fig. 1A).

Hydrogen-rich saline alleviates neuronal necroptosis after TBI. Neuronal necroptosis is the main factor resulting in EBI after TBI (46). Therefore, a TUNEL assay was conducted to examine the degree of cell death in TBI mice treated or non-treated with hydrogen-rich saline $72 \mathrm{~h}$ following model establishment. Neuronal death in the hippocampus decreased upon hydrogen-rich saline treatment compared with the TBI group (Fig. 2A). The expression levels of necroptosis-related proteins were additionally detected via western blotting (Fig. 2B). The results of western blotting also demonstrated that hydrogen-rich saline significantly reduced the expression levels of the necroptosis-related proteins RIP1 and RIP3 compared with the TBI group (Fig. 2C and D). These findings indicated that hydrogen-rich saline exhibited neuroprotective benefits after TBI.

Hydrogen-rich saline alleviates neuroinflammation after TBI. Previous studies have demonstrated that neuroinflammation exhibits a vital function in EBI after TBI and enhanced neuroinflammation can aggravate EBI (10,47-49). Activation of the inflammatory process can induce the release of inflammatory cytokines, which include NF- $\kappa \mathrm{B}$, TNF- $\alpha$ IL- 6 and IL-1 $\beta(39,50)$. Therefore, ELISA was employed to examine the hippocampal levels of NF- $\kappa \mathrm{B}, \mathrm{TNF}-\alpha$ IL- 6 and IL- $1 \beta$. The findings revealed that the inflammatory cytokines were increased substantially after TBI, while they were significantly decreased after hydrogen-rich saline treatment (Fig. 3A-D). Thus, these results suggested that hydrogen-rich saline exhibited a potent anti-inflammatory activity against TBI-induced neuroinflammation.

Hydrogen-rich saline inhibits TBI-induced oxidative stress and decreases ROS levels. To clarify whether oxidative stress performs a crucial function in TBI and whether hydrogen-rich saline can regulate oxidative stress, the oxidative stress-related biomarker levels of GSH, MDA and SOD were detected in each cohort. The findings illustrated that MDA increased following TBI, but considerably decreased following hydrogen-rich saline treatment (Fig. 4A). Additionally, SOD and GSH decreased after TBI but increased considerably following hydrogen-rich saline treatment (Fig. 4B and C). ROS are considered to be a biomarker of oxidative stress activation initiating programmed cell and neuronal death $(31,51)$. ROS levels were detected using the DCF-DA probe. The results demonstrated that ROS levels were increased after TBI in the hippocampus, while decreased after hydrogen-rich saline administration (Figs. 4D and S1).

Hydrogen-rich saline regulates necroptosis via the ROS/HO-1 signaling pathway after TBI. It was explored whether necroptosis inhibition occurred through the ROS/HO-1 signaling pathway after hydrogen-rich saline treatment. The mRNA expression levels of $\mathrm{HO}-1$ and $\mathrm{Nrf} 2$ were detected via RT-qPCR. The findings illustrated that the expression levels of HO-1 and Nrf2 were considerably reduced in the TBI cohort, and were elevated following hydrogen-rich saline administration (Fig. 5A and B). The protein expression levels of HO-1 and $\mathrm{Nrf} 2$ were also detected via western blotting (Fig. 5C). 
A
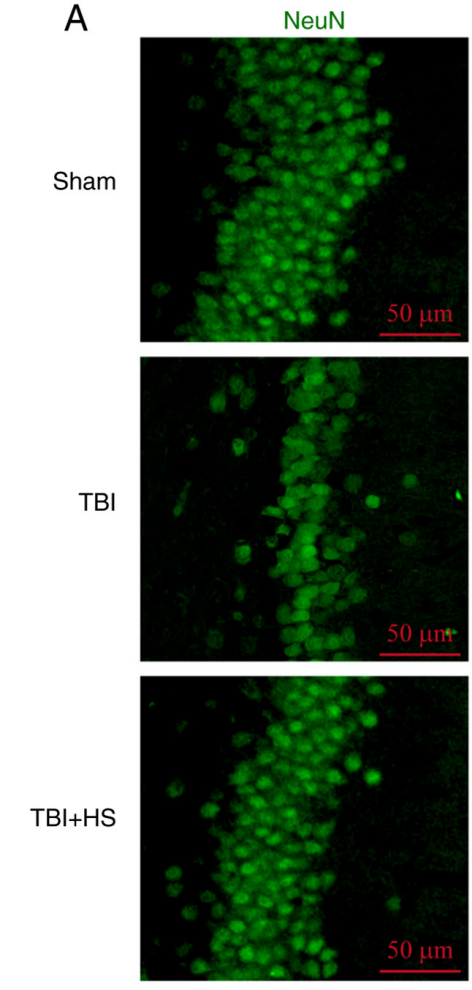

B

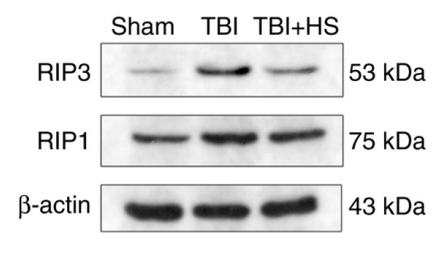

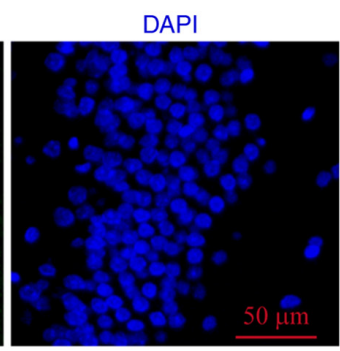
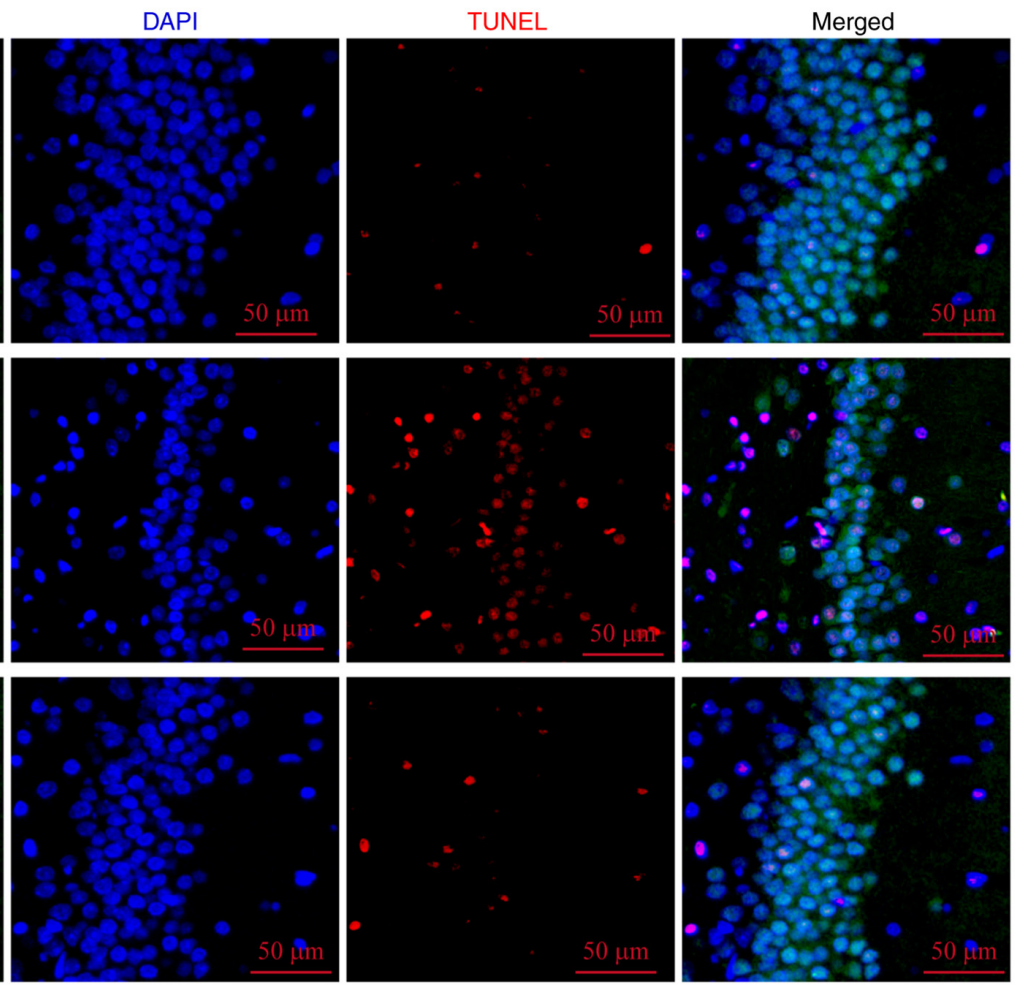

C

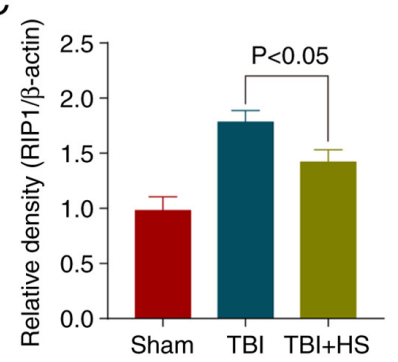

$\mathrm{D}$

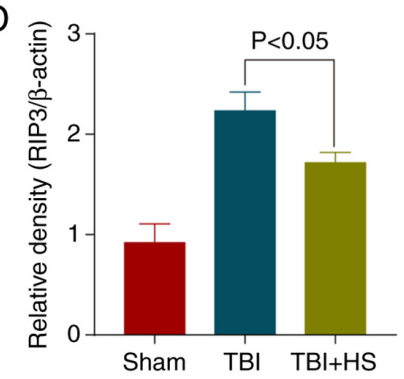

Figure 2. HS alleviates neuronal necroptosis after TBI. (A) TUNEL assay demonstrated that HS alleviated neuronal necroptosis. (B) Expression of RIP1 and RIP3 in the brain cortex of mice following TBI was evaluated via western blotting. Quantification of (C) RIP1 and (D) RIP3 protein levels in the brain cortex relative to $\beta$-actin loading control, indicating that HS suppressed RIP1 and RIP3 expression after TBI in mice (mean \pm SEM; $n=6$ ). TBI, traumatic brain injury; HS, hydrogen-rich saline; RIP, receptor-interacting protein kinase.

Quantification of the protein levels of HO-1 and Nrf2 in the brain cortex relatively to the $\beta$-actin loading control revealed that hydrogen-rich saline increased HO-1 and Nrf2 expression after TBI in mice (Fig. 5D and E). Thus, the present findings collectively demonstrated that hydrogen-rich saline may inhibit TBI-induced necroptosis by regulating the ROS/HO-1 signaling pathway.

\section{Discussion}

The present study examined the therapeutic value of hydrogen-rich saline for ameliorating EBI in a TBI mouse model. The findings indicated that hydrogen-rich saline was a neuroprotective agent that can attenuate EBI following TBI. The results demonstrated that hydrogen-rich saline could achieve the following: i) Ameliorate neurological dysfunction following TBI; ii) relieve brain damage in a mouse TBI model; iii) relieve neuroinflammation after TBI and decrease brain inflammatory damage; and iv) prevent necroptosis after TBI and alleviate neuronal death. Furthermore, the anti-neuroinflammatory and anti-necroptotic roles of hydrogen-rich saline may be associated with the ROS/HO-1 pathway.

Hydrogen-rich saline or hydrogen gas can easily penetrate the BBB via gaseous diffusion, which is acknowledged to achieve protective effects in several CNS disorders, such as TBI, neurodegenerative diseases, intracranial hemorrhage and ischemic stroke (23-26). Hydrogen gas or hydrogen-rich saline serves an important antioxidant role with high tissue transferability, and it has been demonstrated that $\mathrm{H}_{2}$ is safe for patients and animals (37). The anti-oxidative stress and anti-inflammatory effects of hydrogen-rich saline or hydrogen gas are mediated by selective suppression of toxic ROS, which include peroxynitrite and hydroxyl radical (25). Liu et al (52) also reported that $\mathrm{H}_{2}$ can markedly improve cognitive dysfunction and survival rates, decrease inflammatory response and oxidative stress, and increase antioxidant enzyme activities in the serum and hippocampus in a mouse model of sepsis. In the intracerebral hemorrhage model, it has also been demonstrated that hydrogen performs a neuroprotective function 

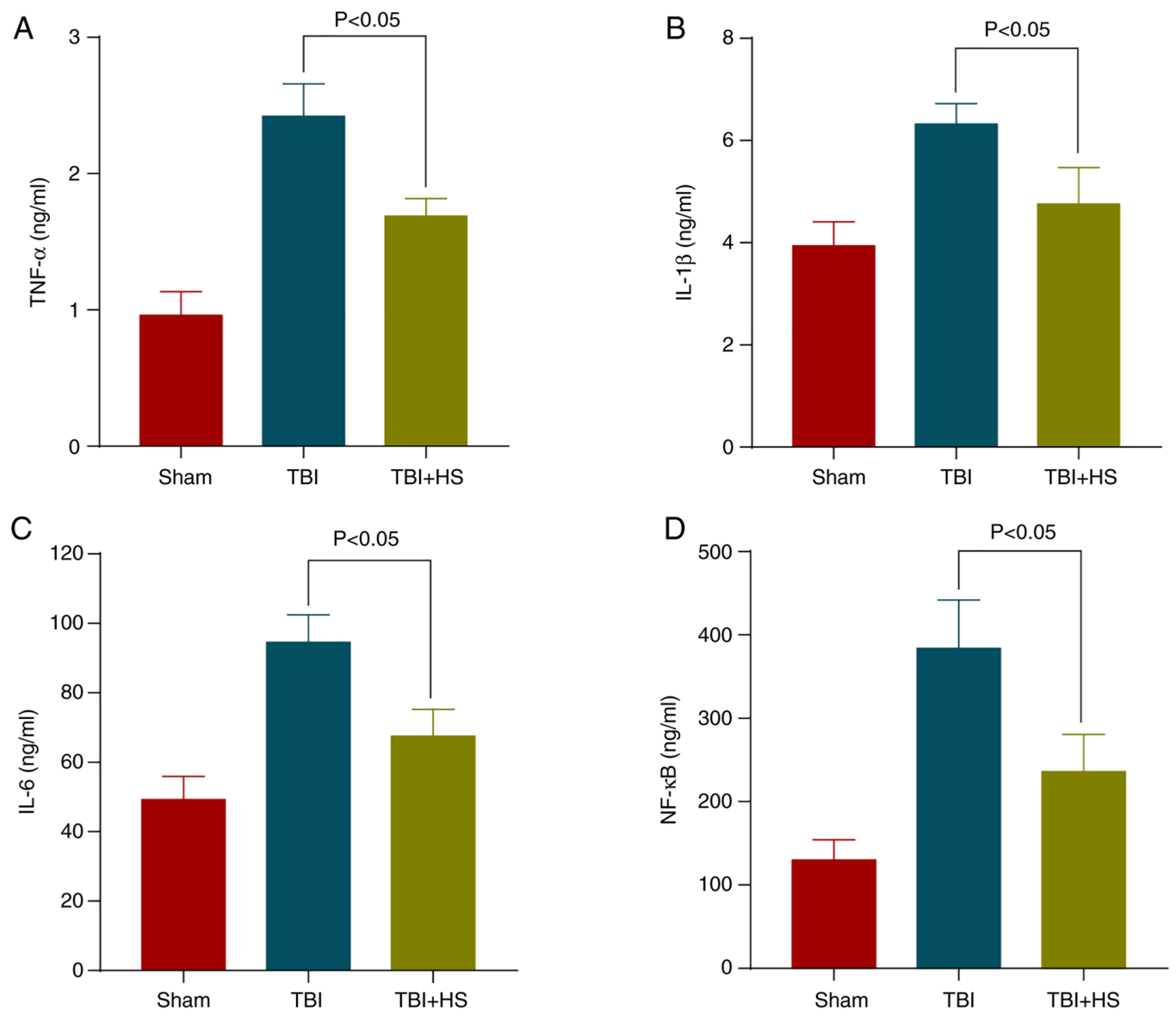

Figure 3. HS alleviates neuroinflammation after TBI. HS significantly decreased the levels of hippocampal (A) TNF- $\alpha$, (B) IL-1 $1 \beta$, (C) IL-6 and (D) NF- $\kappa$ B at $72 \mathrm{~h}$ after TBI (mean $\pm \mathrm{SEM} ; \mathrm{n}=6$ ). TBI, traumatic brain injury; HS, hydrogen-rich saline.
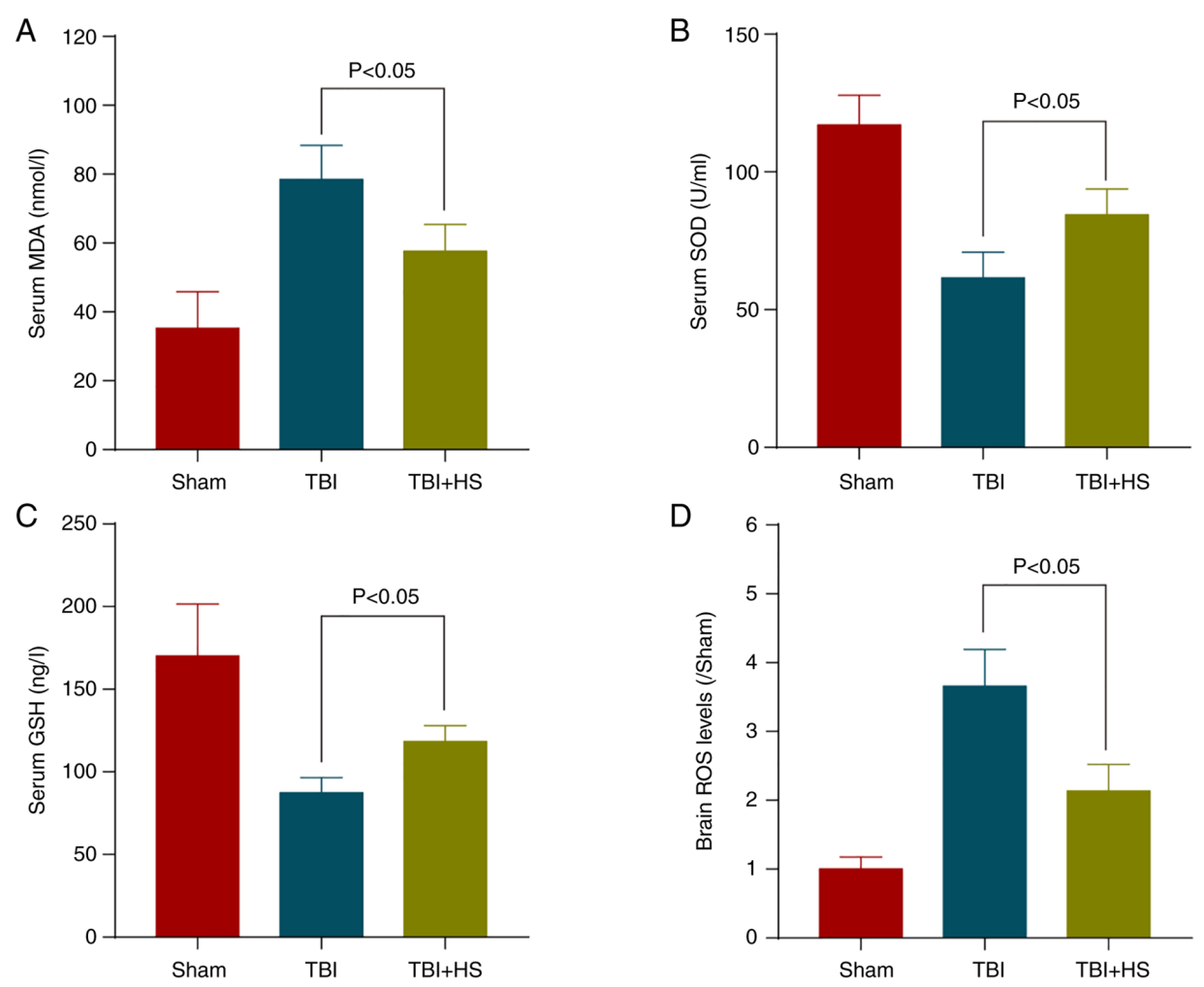

Figure 4. HS inhibits TBI-induced oxidative stress and decreases ROS levels. HS significantly (A) decreased the expression levels of serum MDA, increased the expression levels of (B) serum SOD and (C) serum GSH, and (D) decreased the levels of brain tissue ROS after TBI (mean \pm SEM; $n=6$ ). TBI, traumatic brain injury; HS, hydrogen-rich saline; MDA, malondialdehyde; SOD, superoxide dismutase; GSH, glutathione; ROS, reactive oxygen species. 

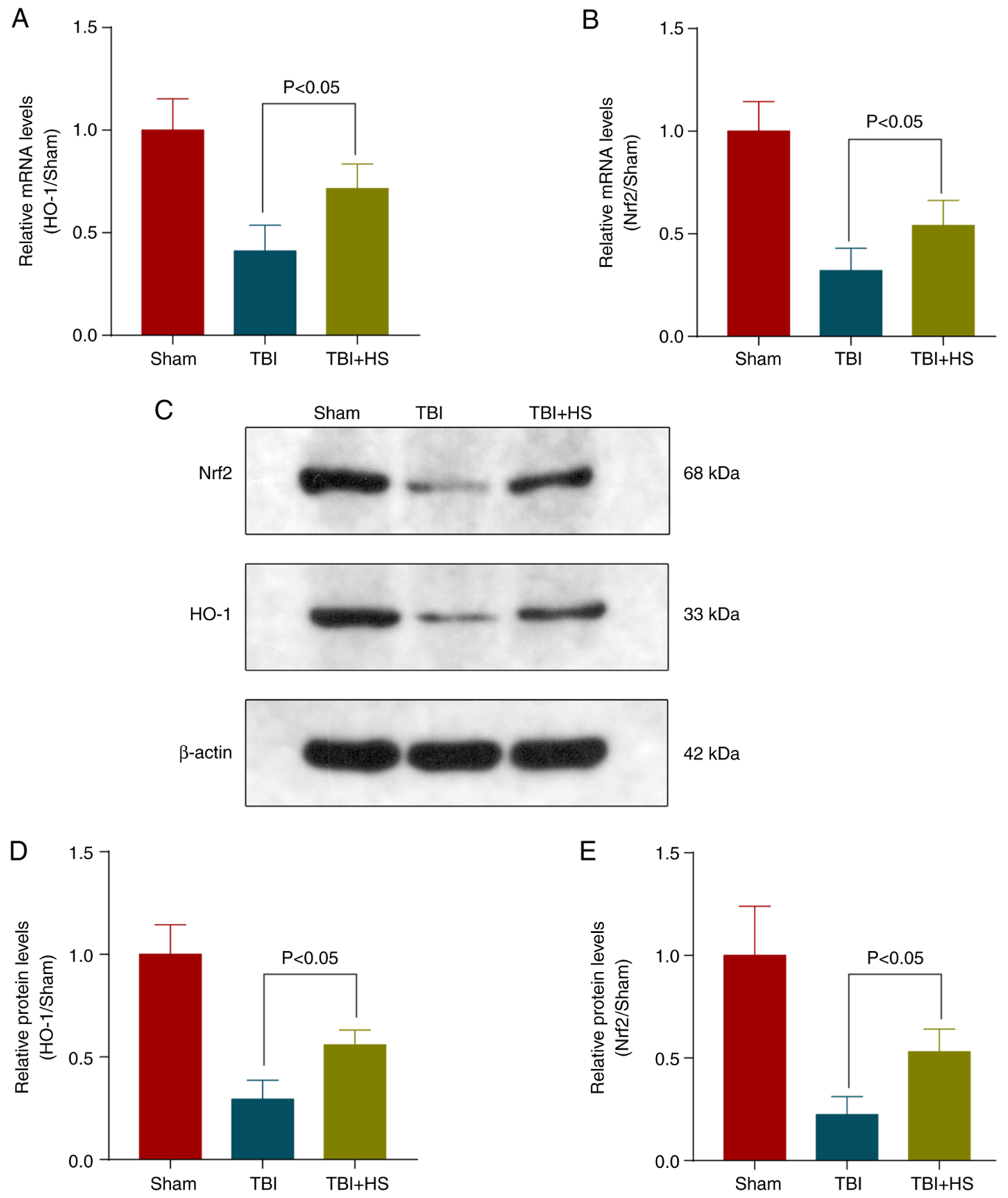

Figure 5. HS regulates necroptosis via the ROS/HO-1 signaling pathway after TBI. (A) HO-1 and (B) Nrf2 mRNA levels in the brain of TBI mice were quantified via reverse transcription-quantitative PCR. (C) Expression of HO-1 and Nrf2 in the brain cortex of mice following TBI was assessed via western blotting. Quantification of (D) HO-1 and (E) Nrf2 protein levels in the brain cortex relative to $\beta$-actin loading control. HS increased HO-1 and Nrf2 expression following TBI in mice. (mean \pm SEM; $n=6$ ). TBI, traumatic brain injury; HS, hydrogen-rich saline; HO-1, heme oxygenase-1; Nrf2, nuclear factor erythroid 2-related factor 2.

against EBI following ICH, alleviating brain edema and neurologic deficits by regulating oxidative stress, neuroinflammation and apoptosis (53). In the hypoxic-ischemic brain injury neonatal rat model, $\mathrm{H}_{2}$ inhalation could alleviate brain damage and improve early neurological outcomes through anti-inflammatory, anti-apoptotic and antioxidant responses via the MAPK/HO-1/peroxisome proliferator-activated receptor $\gamma$ coactivator 1a pathway (54). In the TBI model, molecular hydrogen water could also reverse the controlled cortical impact-induced brain edema through preserved or increased ATP levels (55). Tian et al (56) demonstrated that hydrogen-rich water could alleviate inflammation and decrease brain damage, exhibiting a neuroprotective function against TBI. Yuan et al (57) also discovered that hydrogen-rich water can inhibit oxidative stress and activate the Nrf2 pathway, thereby improving TBI-induced brain damage. Both aforementioned studies explored the neuroprotective effect of hydrogen-rich water associated with inflammation and oxidative stress without investigating further molecular mechanisms. However, the current study also revealed that hydrogen-rich saline considerably enhanced neurological function, alleviated brain edema, increased neuronal survival and downregulated the protein expression of RIP3 and RIP1, as well as the cytokines NF- $\kappa$ B, TNF- $\alpha$, IL- $1 \beta$ and IL-6, following TBI.

Necroptosis is a cell death type of modulated necrosis, requiring the proteins MLKL and RIP3, and is stimulated by death receptors (12). Increasing evidence suggests that necroptosis performs an instrumental function in CNS diseases, such 
as TBI (13-15). Our previous studies have also demonstrated that necroptosis has an integral function in the pathophysiology of neuronal death following in vitro traumatic neuronal injury, and that the potential regulatory mechanisms may be associated with the Akt/GSK-3 $\beta$ and the metabotropic glutamate receptor 1 signaling pathways $(13,14)$. Therefore, necroptosis is a vital mechanism in TBI, and necroptosis inhibition via drugs may decrease neuronal death. Jia et al (58) reported that hydrogen can decrease the expression levels of necroptosis-related proteins in the hippocampus, including MLKL, phosphorylated MLKL and RIP3, thereby partly preventing neuronal and astrocyte necroptosis in the lithium-pilocarpine model of status epilepticus. Dong et al (59) also reported that high concentrations of hydrogen inhalation can alleviate skin ischemia/reperfusion injury by regulating necroptosis and the RIP-MLKL-serine/threonine-protein phosphatase PGAM5/dynamin-related protein 1 necrotic pathway. In the present study, the results confirmed that the mRNA and protein expression levels of RIP3 and RIP1 were elevated $72 \mathrm{~h}$ after TBI, while hydrogen-rich saline treatment could reduce the RIP3 and RIP1 expression levels.

The molecular mechanism of necroptosis and neuroinflammation is complex, and the specific mechanisms of the neuroprotective benefits of hydrogen-rich saline treatment are yet to be elucidated. Wang et al (60) reported that rehmapicrogenin can improve adriamycin-induced nephropathy in vitro and in vivo by reducing ROS accumulation, thereby regulating the expression levels of Nrf2. Nrf2 is an important transcriptional regulation factor that can regulate the expression of $>250$ genes and is characterized by its binding site 'antioxidant response element'; the majority of regulated genes by Nrf2 are involved in oxidative stress and cell apoptosis, necroptosis, autophagy and ferroptosis (31). Yu et al (61) discovered that the inhalation of $2 \%$ molecular hydrogen gas may enhance the survival rates, reduce the lung edema and the lung injury score, and ameliorate the injuries induced by inflammation and oxidative stress in the septic mouse model, while Nrf2 knockout could reverse or weaken the protection of $\mathrm{H}_{2}$ gas on lung damage, which was also dependent on HO-1 and high mobility group protein B1. Additionally, Chen et al (62) demonstrated that $\mathrm{H}_{2}$ decreased endothelial inflammation and injury and increased the expression and activity of HO-1 in vivo and in vitro. Nrf2 knockout or HO-1 inhibition reversed the protection of $\mathrm{H}_{2}$, indicating that this was dependent on the activity of the Nrf2/HO-1 signaling pathway. However, the exact mechanism requires further elucidation.

In summary, the present findings demonstrated that necroptosis, which is mediated by the RIP1 and RIP3 proteins, is a key cellular regulatory mechanism that may lead to EBI following TBI. The present study, for the first time to the best of our knowledge, revealed that hydrogen-rich saline induced modulation of necroptosis and neuroinflammation via the ROS/HO-1 pathway, and also provided a novel insight into evaluating the biological impacts as well as the mechanisms that underly neuroprotection and inhibition of inflammation and necroptosis by hydrogen-rich saline.

\section{Acknowledgements}

Not applicable.

\section{Funding}

No funding was received.

\section{Availability of data and materials}

The datasets used and/or analyzed during the current study are available from the corresponding author on reasonable request.

\section{Authors' contributions}

YH wrote the manuscript. YH, XF, YW, LS and JC performed the experiments and prepared the figures. YH and JC confirm the authenticity of all the raw data. YH and JC designed the study and revised the manuscript. All authors read and approved the final manuscript.

\section{Ethics approval and consent to participate}

The study protocol was approved by the Research Ethics Committee of Wuxi Clinical College of Anhui Medical University (approval no. YXLL-2020-012; Wuxi, China).

\section{Patient consent for publication}

Not applicable.

\section{Competing interests}

The authors declare that they have no competing interests.

\section{References}

1. Jiang JY, Gao GY, Feng JF, Mao Q, Chen LG, Yang XF, Liu JF, Wang YH, Qiu BH and Huang XJ: Traumatic brain injury in China. Lancet Neurol 18: 286-295, 2019.

2. Chen J, Li M, Chen L, Chen W, Zhang C, Feng Y, Wang Y and Chen Q: The effect of controlled decompression for severe traumatic brain injury: A randomized, controlled trial. Front Neurol 11: 107, 2020.

3. Chen JH, Li PP, Yang LK, Chen L, Zhu J, Hu X and Wang YH: Value of ventricular intracranial pressure monitoring for traumatic bifrontal contusions. World Neurosurg 113: e690-e701, 2018.

4. Nichol A, French C, Little L, Haddad S, Presneill J, Arabi Y, Bailey M, Cooper DJ, Duranteau J, Huet O, et al: Erythropoietin in traumatic brain injury (EPO-TBI): A double-blind randomised controlled trial. Lancet 386: 2499-2506, 2015.

5. Hutchinson PJ, Kolias AG, Timofeev IS, Corteen EA, Czosnyka M Timothy J, Anderson I, Bulters DO, Belli A, Eynon CA, et al: Trial of decompressive craniectomy for traumatic intracranial hypertension. N Engl J Med 375: 1119-1130, 2016.

6. Cooper DJ, Nichol AD, Bailey M, Bernard S, Cameron PA, Pili-Floury S, Forbes A, Gantner D, Higgins AM, Huet O, et al: Effect of early sustained prophylactic hypothermia on neurologic outcomes among patients with severe traumatic brain injury: The POLAR randomized clinical trial. JAMA 320: 2211-2220, 2018.

7. Wright DW, Yeatts SD, Silbergleit R, Palesch YY, Hertzberg VS, Frankel M, Goldstein FC, Caveney AF, Howlett-Smith H, Bengelink EM, et al: Very early administration of progesterone for acute traumatic brain injury. N Engl J Med 371: 2457-2466, 2014.

8. Robertson CS, Hannay HJ, Yamal JM, Gopinath S, Goodman JC, Tilley BC; Epo Severe TBI Trial Investigators, Baldwin A, Rivera Lara L, Saucedo-Crespo H, et al: Effect of erythropoietin and transfusion threshold on neurological recovery after traumatic brain injury: A randomized clinical trial. JAMA 312: 36-47, 2014. 
9. Wang Y, Wang L, Hu T, Wang F, Han Z, Yin Z, Ge X, Xie K and Lei P: Hydrogen improves cell viability partly through inhibition of autophagy and activation of PI3K/Akt/GSK3 $\beta$ signal pathway in a microvascular endothelial cell model of traumatic brain injury. Neurol Res 42: 487-496, 2020.

10. Li H, Lu C, Yao W, Xu L, Zhou J and Zheng B: Dexmedetomidine inhibits inflammatory response and autophagy through the circLrp1b/miR-27a-3p/Dram2 pathway in a rat model of traumatic brain injury. Aging (Albany NY) 12: 21687-21705, 2020.

11. Wang Y, Zhao M, Shang L, Zhang Y, Huang C, He Z, Luo M, Wu B, Song P, Wang M and Duan F: Homerla protects against neuronal injury via PI3K/AKT/mTOR signaling pathway. Int J Neurosci 130: 621-630, 2020

12. Vandenabeele P, Galluzzi L, Vanden Berghe T and Kroemer G: Molecular mechanisms of necroptosis: An ordered cellular explosion. Nat Rev Mol Cell Biol 11: 700-714, 2010.

13. Chen T, Yang LK, Zhu J, Hang CH and Wang YH: The AMPAR antagonist perampanel regulates neuronal necroptosis via Akt/GSK3 $\beta$ signaling after acute traumatic injury in cortical neurons. CNS Neurol Disord Drug Targets 20: 266-272, 2021.

14. Chen T, Zhu J, Wang YH and Hang CH: Arc silence aggravates traumatic neuronal injury via mGluR1-mediated ER stress and necroptosis. Cell Death Dis 11: 4, 2020.

15. Bao Z, Fan L, Zhao L, Xu X, Liu Y, Chao H, Liu N, You Y, Liu Y, Wang $X$ and Ji J: Silencing of A20 aggravates neuronal death and inflammation after traumatic brain injury: A potential trigger of necroptosis. Front Mol Neurosci 12: 222, 2019

16. Laird MD, Wakade C, Alleyne $\mathrm{CH}$ Jr and Dhandapani KM Hemin-induced necroptosis involves glutathione depletion in mouse astrocytes. Free Radic Biol Med 45: 1103-1114, 2008.

17. Shen H, Liu C, Zhang D, Yao X, Zhang K, Li H and Chen G: Role for RIP1 in mediating necroptosis in experimental intracerebral hemorrhage model both in vivo and in vitro. Cell Death Dis 8 : e2641, 2017.

18. Zhang Y, Li M, Li X, Zhang H, Wang L, Wu X, Zhang $\mathrm{H}$ and Luo Y: Catalytically inactive RIP1 and RIP3 deficiency protect against acute ischemic stroke by inhibiting necroptosis and neuroinflammation. Cell Death Dis 11: 565, 2020.

19. Yuan J, Amin P and Ofengeim D: Necroptosis and RIPK1mediated neuroinflammation in CNS diseases. Nat Rev Neurosci 20: 19-33, 2019.

20. Liu C, Chen Y, Cui W, Cao Y, Zhao L, Wang H, Liu X, Fan S, Huang K, Tong A and Zhou L: Inhibition of neuronal necroptosis mediated by RIP1/RIP3/MLKL provides neuroprotective effects on kaolin-induced hydrocephalus in mice. Cell Prolif 54: e13108, 2021.

21. Wu Y, Zheng Z, Cao X, Yang Q, Norton V, Adini A, Maiti AK, Adini I and $\mathrm{Wu} \mathrm{H}$ : RIP1/RIP3/MLKL mediates myocardial function through necroptosis in experimental autoimmune myocarditis. Front Cardiovasc Med 8: 696362, 2021

22. Linkermann A and Green DR: Necroptosis. N Engl J Med 370: 455-465, 2014

23. Zou R, Wang MH, Chen Y, Fan X, Yang B, Du J, Wang XB, Liu KX and Zhou J: Hydrogen-rich saline attenuates acute lung injury induced by limb ischemia/reperfusion via down-regulating chemerin and NLRP3 in rats. Shock 52: 134-141, 2019.

24. Ning K, Liu WW, Huang JL, Lu HT and Sun XJ: Effects of hydrogen on polarization of macrophages and microglia in a stroke model. Med Gas Res 8: 154-159, 2019.

25. Kumagai K, Toyooka T, Takeuchi S, Otani N, Wada K, Tomiyama A and Mori K: Hydrogen gas inhalation improves delayed brain injury by alleviating early brain injury after experimental subarachnoid hemorrhage. Sci Rep 10: 12319, 2020

26. Ohno K, Ito M, Ichihara M and Ito M: Molecular hydrogen as an emerging therapeutic medical gas for neurodegenerative and other diseases. Oxid Med Cell Longev 2012: 353152, 2012.

27. Takeuchi S, Mori K, Arimoto H, Fujii K, Nagatani K, Tomura S, Otani N, Osada $\mathrm{H}$ and Wada K: Effects of intravenous infusion of hydrogen-rich fluid combined with intra-cisternal infusion of magnesium sulfate in severe aneurysmal subarachnoid hemorrhage: Study protocol for a randomized controlled trial. BMC Neurol 14: 176, 2014.

28. Schallner N, Pandit R, LeBlanc R III, Thomas AJ, Ogilvy CS, Zuckerbraun BS, Gallo D, Otterbein LE and Hanafy KA Microglia regulate blood clearance in subarachnoid hemorrhage by heme oxygenase-1. J Clin Invest 125: 2609-2625, 2015.

29. Kaiser S, Frase S, Selzner L, Lieberum JL, Wollborn J, Niesen WD, Foit NA, Heiland DH and Schallner N: Neuroprotection after hemorrhagic stroke depends on cerebral heme oxygenase-1. Antioxidants (Basel) 8: 496, 2019.
30. Afonso MB, Rodrigues PM, Simão AL, Ofengeim D, Carvalho T, Amaral JD, Gaspar MM, Cortez-Pinto H, Castro RE, Yuan J and Rodrigues CM: Activation of necroptosis in human and experimental cholestasis. Cell Death Dis 7: e2390, 2016.

31. Chen J, Wang Y, Wu J, Yang J, Li M and Chen Q: The potential value of targeting ferroptosis in early brain injury after acute CNS disease. Front Mol Neurosci 13: 110, 2020.

32. National Research Council (US). Committee for the Update of the Guide for the Care and Use of Laboratory Animals: The National Academies Collection: Reports funded by National Institutes of Health. In: Guide for the Care and Use of Laboratory Animals. 8th edition. National Academies Press, Washington, DC, 2011.

33. Flierl MA, Stahel PF, Beauchamp KM, Morgan SJ, Smith WR and Shohami E: Mouse closed head injury model induced by a weight-drop device. Nat Protoc 4: 1328-1337, 2009.

34. Tian J, Yang L, Wang P, Yang L and Fan Z: Exogenous CGRP regulates apoptosis and autophagy to alleviate traumatic brain injury through Akt/mTOR signalling pathway. Neurochem Res 45: 2926-2938, 2020

35. Zhuang Z, Zhou ML, You WC, Zhu L, Ma CY, Sun XJ and Shi JX: Hydrogen-rich saline alleviates early brain injury via reducing oxidative stress and brain edema following experimental subarachnoid hemorrhage in rabbits. BMC Neurosci 13 47, 2012.

36. Feng Y, Wang R, Xu J, Sun J, Xu T, Gu Q and Wu X: Hydrogen-rich saline prevents early neurovascular dysfunction resulting from inhibition of oxidative stress in STZ-diabetic rats. Curr Eye Res 38: 396-404, 2013.

37. Ohsawa I, Ishikawa M, Takahashi K, Watanabe M, Nishimaki K, Yamagata K, Katsura K, Katayama Y, Asoh S and Ohta S: Hydrogen acts as a therapeutic antioxidant by selectively reducing cytotoxic oxygen radicals. Nat Med 13: 688-694, 2007.

38. Tang C, Shan Y, Hu Y, Fang Z, Tong Y, Chen M, Wei X, Fu X and $\mathrm{Xu} X$ : FGF2 attenuates neural cell death via suppressing autophagy after rat mild traumatic brain injury. Stem Cells Int 2017: 2923182, 2017.

39. Chen J, Zhang C, Yan T, Yang L, Wang Y, Shi Z, Li M and Chen Q: Atorvastatin ameliorates early brain injury after subarachnoid hemorrhage via inhibition of pyroptosis and neuroinflammation. J Cell Physiol 236: 6920-6931, 2021.

40. Chen JH, Wu T, Xia WY, Shi ZH, Zhang CL, Chen L, Chen QX and Wang YH: An early neuroprotective effect of atorvastatin against subarachnoid hemorrhage. Neural Regen Res 15: 1947-1954, 2020.

41. Chen J, Xuan Y, Chen Y, Wu T, Chen L, Guan H, Yang S, He J, Shi D and Wang Y: Netrin-1 alleviates subarachnoid haemorrhage-induced brain injury via the PPAR $\gamma / \mathrm{NF}-\mathrm{KB}$ signalling pathway. J Cell Mol Med 23: 2256-2262, 2019.

42. Hollingshead JR and Phillips RK: Haemorrhoids: Modern diagnosis and treatment. Postgrad Med J 92: 4-8, 2016.

43. Das S, Chattopadhyay D, Chatterjee SK, Mondal SA, Majumdar SS, Mukhopadhyay S, Saha N, Velayutham R, Bhattacharya $S$ and Mukherjee S: Increase in PPAR $\gamma$ inhibitory phosphorylation by Fetuin-A through the activation of Ras-MEK-ERK pathway causes insulin resistance. Biochim Biophys Acta Mol Basis Dis 1867: 166050, 2021.

44. Li Y, Liu Y, Wu P, Tian Y, Liu B, Wang J, Bihl J and Shi H: Inhibition of ferroptosis alleviates early brain injury after subarachnoid hemorrhage in vitro and in vivo via reduction of lipid peroxidation. Cell Mol Neurobiol 41: 263-278, 2021.

45. Livak KJ and Schmittgen TD: Analysis of relative gene expression data using real-time quantitative PCR and the 2(-Delta Delta $\mathrm{C}(\mathrm{T})$ ) method. Methods 25: 402-408, 2001

46. Wehn AC, Khalin I, Duering M, Hellal F, Culmsee C, Vandenabeele P, Plesnila N and Terpolilli NA: RIPK1 or RIPK3 deletion prevents progressive neuronal cell death and improves memory function after traumatic brain injury. Acta Neuropathol Commun 9: 138, 2021

47. Huang GR and Hao FG: Dexmedetomidine inhibits inflammation to alleviate early neuronal injury via TLR4/NF- $\mathrm{B}$ pathway in rats with traumatic brain injury. Crit Rev Eukaryot Gene Expr 31: 41-47, 2021.

48. Li F, Wang X, Zhang Z, Zhang X and Gao P: Dexmedetomidine attenuates neuroinflammatory-induced apoptosis after traumatic brain injury via Nrf2 signaling pathway. Ann Clin Transl Neurol 6: 1825-1835, 2019.

49. Yang T, Feng X, Zhao Y, Zhang H, Cui H, Wei M, Yang H and Fan $\mathrm{H}$ : Dexmedetomidine enhances autophagy via $\alpha 2-\mathrm{AR} / \mathrm{AMPK} / \mathrm{mTOR}$ pathway to inhibit the activation of NLRP3 inflammasome and subsequently alleviates lipopolysaccharide-induced acute kidney injury. Front Pharmacol 11: 790, 2020. 
50. Fei W, Jiao W, Feng X, Chen X and Wang Y: Intermittent hypoxia mimicking obstructive sleep apnea aggravates early brain injury following ICH via neuroinflammation and apoptosis. Mol Med Rep 24: 824, 2021.

51. Feng X, Ma W, Zhu J, Jiao W and Wang Y: Dexmedetomidine alleviates early brain injury following traumatic brain injury by inhibiting autophagy and neuroinflammation through the ROS/Nrf2 signaling pathway. Mol Med Rep 24: 661,2021.

52. Liu L, Xie K, Chen H, Dong X, Li Y, Yu Y, Wang G and Yu Y: Inhalation of hydrogen gas attenuates brain injury in mice with cecal ligation and puncture via inhibiting neuroinflammation, oxidative stress and neuronal apoptosis. Brain Res 1589: 78-92, 2014.

53. Choi KS, Kim HJ, Do SH, Hwang SJ and Yi HJ: Neuroprotective effects of hydrogen inhalation in an experimental rat intracerebral hemorrhage model. Brain Res Bull 142: 122-128, 2018.

54. Wang P, Zhao M, Chen Z, Wu G, Fujino M, Zhang C, Zhou W, Zhao M, Hirano SI, Li XK and Zhao L: Hydrogen gas attenuates hypoxic-ischemic brain injury via regulation of the MAPK/HO-1/PGC-1a pathway in neonatal rats. Oxid Med Cell Longev 2020: 6978784, 2020.

55. Dohi K, Kraemer BC, Erickson MA, McMillan PJ, Kovac A, Flachbartova Z, Hansen KM, Shah GN, Sheibani N, Salameh T and Banks WA: Molecular hydrogen in drinking water protects against neurodegenerative changes induced by traumatic brain injury. PLoS One 9: e108034, 2014.

56. Tian R, Hou Z, Hao S, Wu W, Mao X, Tao X, Lu T and Liu B: Hydrogen-rich water attenuates brain damage and inflammation after traumatic brain injury in rats. Brain Res 1637: 1-13, 2016.
57. Yuan J, Wang D, Liu Y, Chen X, Zhang H, Shen F, Liu X and Fu J: Hydrogen-rich water attenuates oxidative stress in rats with traumatic brain injury via Nrf2 pathway. J Surg Res 228: 238-246, 2018.

58. Jia R, Jia N, Yang F, Liu Z, Li R, Jiang Y, Zhao J, Wang L, Zhang S, Zhang Z, et al: Hydrogen alleviates necroptosis and cognitive deficits in lithium-pilocarpine model of status epilepticus. Cell Mol Neurobiol 39: 857-869, 2019.

59. Dong XH,Liu H,Zhang MZ,Zhao PX, Liu S, Hao Y and Wang YB: Postconditioning with inhaled hydrogen attenuates skin ischemia/reperfusion injury through the RIP-MLKL-PGAM5/Drp1 necrotic pathway. Am J Transl Res 11: 499-508, 2019.

60. Wang M, Ke Y, Li Y, Shan Z, Mi W, Cao Y, Feng W and Zheng X: The nephroprotective effects and mechanisms of rehmapicrogenin include ROS inhibition via an oestrogen-like pathway both in vivo and in vitro. Biomed Pharmacother 138: 111305, 2021.

61. Yu Y, Yang Y, Yang M, Wang C, Xie K and Yu Y: Hydrogen gas reduces HMGB1 release in lung tissues of septic mice in an Nrf2/HO-1-dependent pathway. Int Immunopharmacol 69: 11-18, 2019.

62. Chen H, Xie K, Han H, Li Y, Liu L, Yang T and Yu Y: Molecular hydrogen protects mice against polymicrobial sepsis by ameliorating endothelial dysfunction via an $\mathrm{Nrf} 2 / \mathrm{HO}-1$ signaling pathway. Int Immunopharmacol 28: 643-654, 2015.

This work is licensed under a Creative Commons Attribution-NonCommercial-NoDerivatives 4.0 International (CC BY-NC-ND 4.0) License. 